\title{
Two-dome structure in electron-doped iron arsenide superconductors
}

Soshi limura ${ }^{1}$, Satoru Matsuishi ${ }^{1}$, Hikaru Sato ${ }^{1}$, Taku Hanna ${ }^{1}$, Yoshinori Muraba ${ }^{1}$, Sung Wng Kim ${ }^{2, \dagger}$, Jung Eun $\mathrm{Kim}^{3}$, Masaki Takata ${ }^{3,4} \&$ Hideo Hosono ${ }^{1,2}$

Iron arsenide superconductors based on the material $\mathrm{LaFeAsO}_{1-x} \mathrm{~F}_{x}$ are characterized by a twodimensional Fermi surface (FS) consisting of hole and electron pockets yielding structural and antiferromagnetic transitions at $x=0$. Electron doping by substituting $\mathrm{O}^{2-}$ with $\mathrm{F}^{-}$suppresses these transitions and gives rise to superconductivity with a maximum $T_{\mathrm{c}}$ of $26 \mathrm{~K}$ at $x=0.1$. However, the over-doped region cannot be accessed due to the poor solubility of $\mathrm{F}^{-}$above $x=0.2$. Here we overcome this problem by doping $\mathrm{LaFeAsO}$ with hydrogen. We report the phase diagram of $\mathrm{LaFeAsO}_{1-x} \mathrm{H}_{x}(x<0.53)$ and, in addition to the conventional superconducting dome seen in $\mathrm{LaFeAsO}_{1-x} \mathrm{~F}_{x}$, we find a second dome in the range $0.21<x<0.53$, with a maximum $T_{c}$ of $36 \mathrm{~K}$ at $x=0.3$. Density functional theory calculations reveal that the three Fe $3 d$ bands ( $x y$, $y z$ and $z x$ ) become degenerate at $x=0.36$, whereas the FS nesting is weakened monotonically with $x$. These results imply that the band degeneracy has an important role to induce high $T_{\mathrm{c}}$.

\footnotetext{
${ }^{1}$ Materials and Structures Laboratory, Tokyo Institute of Technology, 4259 Nagatsuta-cho, Midori-ku, Yokohama 226-8503, Japan. ${ }^{2}$ Frontier Research Center, Tokyo Institute of Technology, 4259 Nagatsuta-cho, Midori-ku, Yokohama 226-8503, Japan. ${ }^{3}$ Japan Synchrotron Radiation Research Institute, 1-1-1 Kouto, Sayo-cho, Sayo-gun, Hyogo 679-5198, Japan. ${ }^{4}$ RIKEN SPring-8 Center, 1-1-1 Kouto, Sayo-cho, Sayo-gun, Hyogo 679-5148, Japan. †Present address: Department of Energy Science, Sungkyunkwan University, 300 Cheoncheon, Jangan-ku, Suwon, Korea. Correspondence and request for materials should be addressed to H.H. (e-mail: hosono@lucid.msl.titech.ac.jp).
} 
S ince the discovery of superconductivity in $\mathrm{LaFeAsO}_{1-x} \mathrm{~F}_{x}$ with $T_{\mathrm{c}}=26 \mathrm{~K}$ in early 2008 (ref. 1), various types of iron pnictides containing square lattices of $\mathrm{Fe}^{2+}$ have been investigated ${ }^{2-4}$. That maximum was raised to $55 \mathrm{~K}$ in $L n$-1111-type $\operatorname{LnFeAs} \mathrm{O}_{1-x} \mathrm{~F}_{x}$ ( $\mathrm{Ln}$ denotes lanthanide) ${ }^{5}$. The compound of $\mathrm{LaFeAsO}_{1-x} \mathrm{~F}_{x}$ is paramagnetic metal with tetragonal symmetry at room temperature and undergoes a tetragonal-orthorhombic transition around $150 \mathrm{~K}$ accompanied by a para-antiferromagnetic (AFM) transition ${ }^{6,7}$. Superconductivity emerges when the transitions are suppressed by carrier doping via element substitution or pressure application. To explain the emergence of superconductivity near the AFM phase, a spin fluctuation model resulting from Fermi surface (FS) nesting between hole and electron pockets was proposed based on density functional theory (DFT) calculations ${ }^{8,9}$. This model explains the suppression of superconductivity in $\mathrm{LaFeAsO}_{1-} \mathrm{F}_{x}$ upon electron doping to the filling level of hole pockets $(x=0.2)$ and the striking difference in the maximum $T_{c}$ between $\operatorname{LnFeAsO} \mathrm{O}_{1-x} \mathrm{~F}_{x}(26-55 \mathrm{~K})$ and LaFePO $(4 \mathrm{~K})$ or between La-1111(26 K) and Sm-1111(55 K) ${ }^{10}$.

However, the phase diagrams reported so far for $L n F e A s O$ systems are rather incomplete. For instance, the suppression of $T_{\mathrm{c}}$ in over-doping region had not been confirmed for any Ln-1111-types (except La). This situation primarily comes from the low solubility limit of fluorine in $\operatorname{LnFeAsO} \mathrm{F}_{1-x} \mathrm{~F}_{x}(x<0.15-0.20)$. Recently, we reported the syntheses of $(\mathrm{Ce}, \mathrm{Sm}) \mathrm{FeAsO}_{1-x} \mathrm{H}_{x}(0<x<0.5)$ by using the high solubility limit of hydrogen and obtained a complete superconducting dome ranging $0.05<x \leq 0.4 \sim 0.5$ with optimum $T_{\mathrm{C}}$ of $47 \mathrm{~K}$ for the Ce-system or $56 \mathrm{~K}$ for the Sm-system, agreeing well with that the previous data of each fluorine-doped sample in $x<0.15$ (refs 11,12). The position and occupancy of hydrogen substituting the oxygen sites were verified by neutron powder diffraction measurement and the charge state of hydrogen was examined by DFT calculations $^{12}$. The neutron powder diffraction measurement on $\mathrm{CeFeAsO}_{1-x} \mathrm{D}_{x}$ revealed that hydrogen species exclusively substitute the oxygen sites in the CeO layer, and DFT calculations indicated that the hydrogen $1 s$ band is located at -3 to $-6 \mathrm{eV}$, which is close to level of the oxygen $2 p$ band and the charge state of the hydrogen is -1 . These results substantiate the idea that hydrogen exclusively substituting the $\mathrm{O}^{2-}$ sites occurs as $\mathrm{H}^{-}$, supplying electrons to the FeAs layer, the same as fluorine does $\left(\mathrm{O}^{2-}=\mathrm{H}^{-}+\mathrm{e}^{-}\right)$.
When comparing the superconducting dome of $\mathrm{LaFeAsO}_{1-x} \mathrm{~F}_{x}$ with $(\mathrm{Ce}, \mathrm{Sm}) \mathrm{FeAsO}_{1-x} \mathrm{H}_{x}$, it is found that the dome width is twice as narrow as that of $(\mathrm{Ce}, \mathrm{Sm}) \mathrm{FeAsO}_{1-x} \mathrm{H}_{x}$ and the optimum $T_{\mathrm{c}}$ of $\mathrm{LaFeAsO}_{1-x} \mathrm{~F}_{x}$ is much lower than that of $(\mathrm{Ce}, \mathrm{Sm}) \mathrm{FeAsO}_{1-x} \mathrm{H}_{x}$. Moreover, the temperature dependence of resistivity in the normal conducting state indicates that the $(\mathrm{Ce}, \mathrm{Sm}) \mathrm{FeAsO}_{1-x} \mathrm{H}_{x}$ behave non-Fermi liquid, that is, $\rho(T) \sim T$, whereas $\mathrm{LaFeAsO}_{1-x} \mathrm{~F}_{x}$ obeys Fermi liquid, that is, $\rho(T) \sim T^{2}$. These differences remind us the idea that the electron doping via fluorine substitution in $\mathrm{LaFeAsO}_{1-x} \mathrm{~F}_{x}$ is not enough to draw out the genuine physical property of $\mathrm{LaFeAsO}$.

In this Article, we study the LaFeAsO system, the prototype material for the $L n-1111$ system, and examine its superconducting properties by using $\mathrm{H}^{-}$in place of $\mathrm{F}^{-}$for electron doping. We report the existence over a wider $x$ range of another $T_{\mathrm{c}}$ dome with higher $T_{\mathrm{c}}$ in addition to the dome reported so far by $\mathrm{F}^{-}$substitution. Not only are there differences in maximum $T_{c}$ and shape between these two domes, but also the second dome with resistivity characterized by a linear temperature dependence corresponds to that observed in other $L n-1111$ systems (except La) with higher $T_{c}$. Based on DFT calculations of the crystal structures of these doped samples determined at $20 \mathrm{~K}$, we discuss the origin for the superconductivity.

\section{Results}

Two-dome structure. In Fig. 1a,b, we show the temperature dependence of electrical resistivity for $\mathrm{LaFeAsO}_{1-x} \mathrm{H}_{x}$. At $x=0.01$ and 0.04 , a kink in resistivity due to structural or magnetic transitions was seen around $150 \mathrm{~K}$ (refs 6,7). As $x$ is increased, the transitions are suppressed and the onset $T_{\mathrm{c}}$ appears for $x \geq 0.04$, and zero resistivity is attained for $x \geq 0.08$. The onset $T_{\mathrm{c}}$, determined from the intersection of the two extrapolated lines in Fig. 1c,d attains a maximum of $29 \mathrm{~K}$ at $x=0.08$ and decreases to $18 \mathrm{~K}$ at $x=0.21$, forming the first $T_{\mathrm{c}}(x)$ dome. For $0.08 \leq x \leq 0.21$, the temperature dependence of resistivity $\rho(T)$ in the normal state obeys a $T^{2}$-law, indicating that the system is a strongly correlated metal in Fermi liquid theory ${ }^{13}$. The $T_{\mathrm{c}}(x)$ and temperature dependence of $\rho$ above $T_{\mathrm{c}}$ agree well with those in $\mathrm{LaFeAsO}_{1-x} \mathrm{~F}_{x}$ (ref. 1). Further electron doping $(x>0.21)$ continuously enhances $T_{\mathrm{c}}$ to $36 \mathrm{~K}$ around $x=0.36$ for which $\rho(T)$ exhibits $T$-linear dependence. Figure 1e,f shows the temperature dependence of volume magnetic susceptibility $\chi$
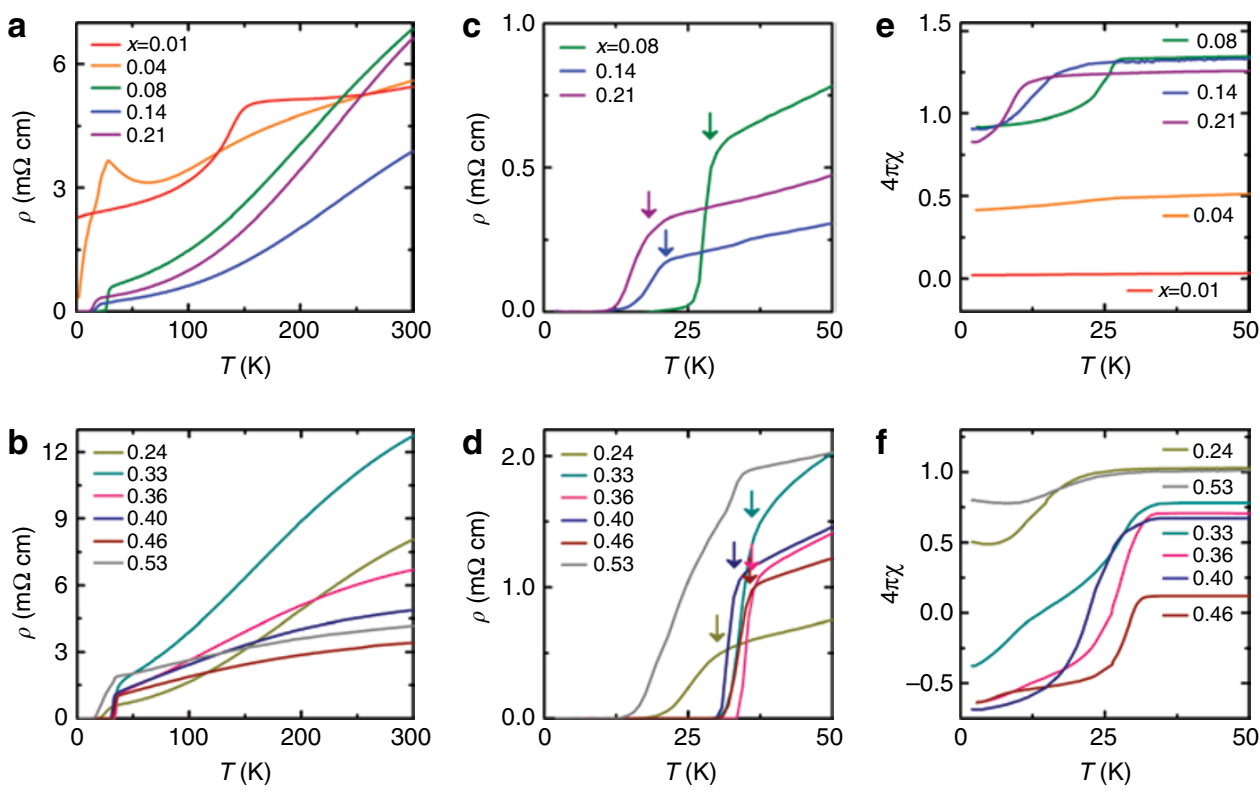

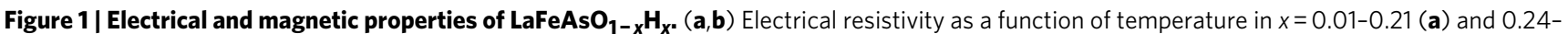
0.53 (b). (c,d) Enlarged $\rho-T$ curves near $T_{c}$ of $\mathrm{LaFeAsO}_{1-x} \mathrm{H}_{x}$ with $x=0.08-0.21$ (c) and 0.24-0.53 (d). The arrows mark the onset $T_{\mathrm{c}}$. (e,f) Magnetic susceptibility data in $x=0.01-0.21(\mathbf{e})$ and 0.24-0.53 (f) measured with a zero-field-cooling history and a field of 10 Oe. 

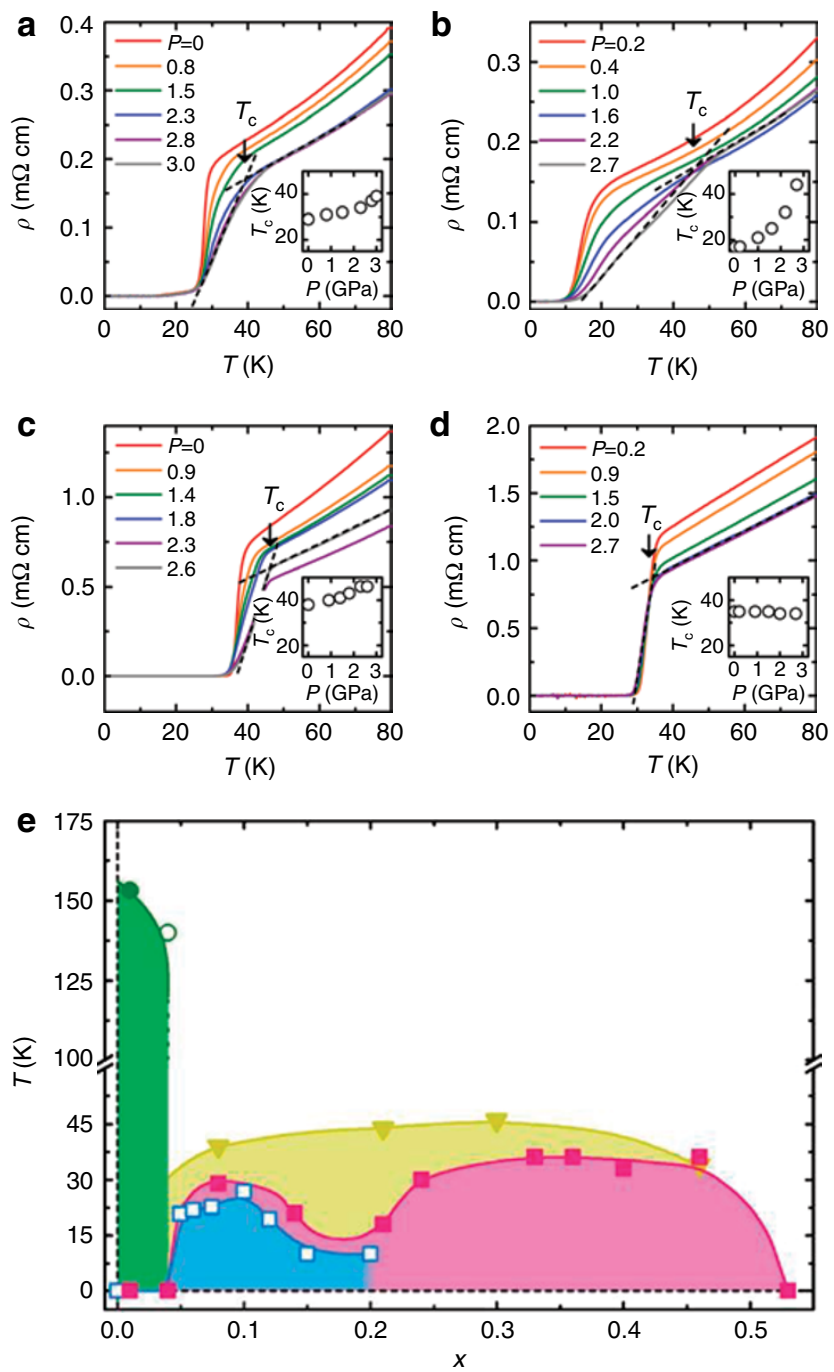

Figure 2 | Temperature dependence of the electrical resistivity of LaFeAsO $_{1-x} \mathrm{H}_{x}$ below $3 \mathrm{GPa}$ and phase diagrams of $\mathrm{LaFeAsO}_{1-x}(\mathrm{H}, \mathrm{F})_{\mathrm{X}}$. (a-d) Temperature dependence of resistivity as a function of static pressure at $x=0.08(\mathbf{a}), 0.21(\mathbf{b}), 0.30(\mathbf{c})$ and $0.46(\mathbf{d})$. The insets show pressure dependence of $T_{\mathrm{c}}$. (e) Electronic phase diagram for $\mathrm{LaFeAsO}_{1-x} \mathrm{H}_{x}$ (filled symbols) and $\mathrm{LaFeAsO}_{1-x} \mathrm{~F}_{x}$ (ref. 15; open symbols). The $T_{\mathrm{c}}$ under ambient (squares) and $3 \mathrm{GPa}$ (inverted triangles) was determined from the intersection of the two extrapolated lines around superconducting transition and $T_{\mathrm{s}}$ (circles) was taken as the anomaly kink in the $\rho-T$ curve ${ }^{7}$.

for samples with different $x$. Because of the presence of metal iron impurities, each sample has a positive offset. The diamagnetism due to superconductivity is clear to see for $x>0.04$. Shielding volume fraction exceeds $40 \%$ at $2 \mathrm{~K}$ in $0.08 \leq x \leq 0.46$, and then decreases to $20 \%$ at $x=0.53$, forming the second $T_{\mathrm{c}}$ dome in the composition range $0.21<x<0.53$. This dome is newly found by the present study.

Pressure effect. Figure $2 \mathrm{a}-\mathrm{d}$ shows the changes in $\rho(T)$ for samples with $x=0.08,0.21,0.30$ and 0.46 under applied pressures $(P)$ up to $3 \mathrm{GPa}$. The onset $T_{\mathrm{c}}$ increases largely with $P$ for $x=0.08,0.21$ and 0.30 , whereas the $T_{\mathrm{c}}$ for $x=0.46$ slightly decreases from $33 \mathrm{~K}$ to $32 \mathrm{~K}$ at $P=2.7 \mathrm{GPa}$. The maximum $T_{\mathrm{c}}$ obtained at $x=0.30$ under $P=2.6 \mathrm{GPa}$ is $46 \mathrm{~K}$, which is distinctly higher than the maximum $T_{\mathrm{c}}$ $(43 \mathrm{~K})$ in the $\mathrm{LaFeAsO}_{1-x} \mathrm{~F}_{x}$ under high pressure ${ }^{14}$. The $T_{\mathrm{c}}$ valley
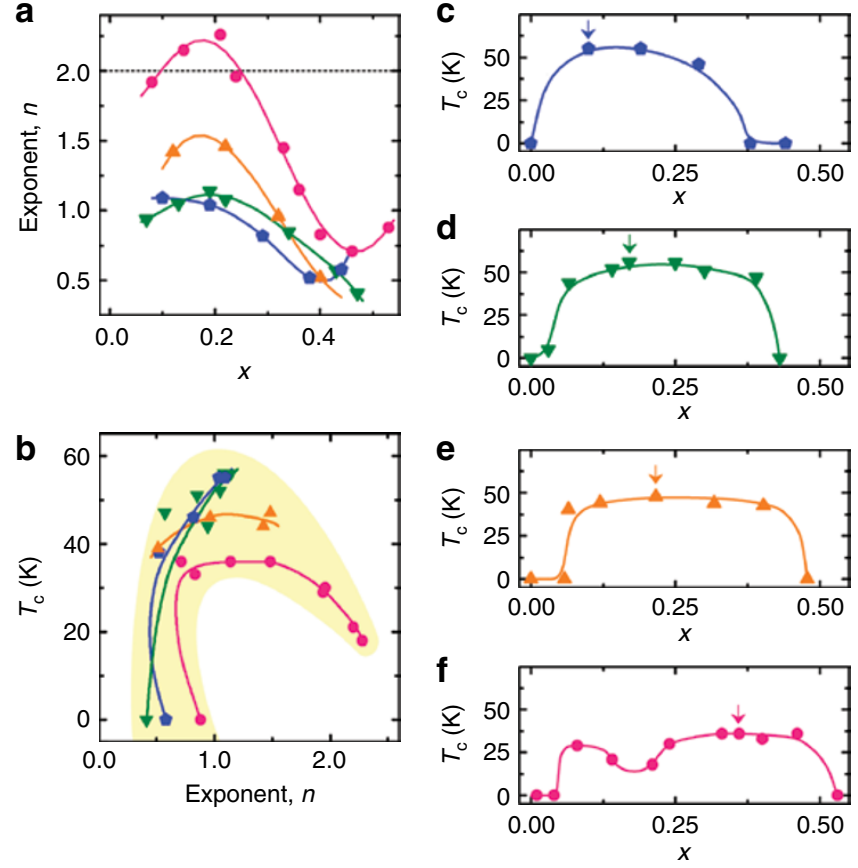

Figure 3 | Effect of lanthanide cation. (a) Relation between the exponent $n$ and $x$ in $\mathrm{LnFeAsO}_{1-x} \mathrm{H}_{x}$ ( $\mathrm{Ln}=\mathrm{La}$ (pink circles), Ce (yellow triangles), $\mathrm{Sm}$ (green inverted triangles) and $\mathrm{Gd}$ (blue pentagons)). The exponent $n$ was obtained by fitting the power-law form $\rho=\rho_{0}+A T^{n}$. The fitting was performed for a linear region in $\log \left[\rho(T)-\rho_{0}\right]$ versus $\log T$ plot. If the shielding volume fraction is $<20 \%$, we set the $T_{C}$ as $0 \mathrm{~K}$ to represent an over-doped region. The dashed line denotes Fermi liquid state $(n=2)$. (b) $T_{\mathrm{C}}$ versus power-law exponent $n$ of $L n \mathrm{FeAsO}_{1-x} \mathrm{H}_{x}(\mathrm{Ln}=\mathrm{La}$ (pink circles), Ce (yellow triangles), Sm (green inverted triangles) and Gd (blue pentagons)). (c-f) Variation in the $T_{\mathrm{c}}$-dome in $\mathrm{LnFeAsO}_{1-x} \mathrm{H}_{x}$ with $L n=\mathrm{La}(\mathbf{f}), \mathrm{Ce}(\mathbf{e}), \mathrm{Sm}(\mathbf{d})$ and $\mathrm{Gd}(\mathbf{c})$. The arrows mark the optimal $T_{\mathrm{c}}$.

around $x=0.21$ under ambient pressure disappears, resulting in the one-dome structure as observed in other $L n-1111$ series. Figure 2e summarizes the $T_{\mathrm{s}}$ and $T_{\mathrm{c}}$ under ambient pressure in the $\mathrm{LaFeAsO}_{1-x} \mathrm{H}_{x}$ and $\mathrm{LaFeAsO}_{1-x} \mathrm{~F}_{x}$ (ref. 15) along with $T_{\mathrm{c}}$ at $P=3 \mathrm{GPa}$ of $\mathrm{LaFeAsO}_{1-x} \mathrm{H}_{x}$. Two superconducting domes are evident and each has maximum $T_{\mathrm{c}}$ around $x=0.08$ and 0.36 . The first dome is located adjacent to the orthorhombic and AFM phase and is almost the same as previously reported for $\mathrm{LaFeAsO}_{1-x} \mathrm{~F}_{x}$, whereas the second dome appears adjacent to the first dome. At $P=3 \mathrm{GPa}$, the two domes merge into a wider dome having a closed shape and a range similar to those in $\mathrm{CeFeAsO}_{1-x} \mathrm{H}_{x}$ with maximum $T_{\mathrm{c}}=47 \mathrm{~K}$ (ref. 12). This unification of the two domes on applying high pressure may be understood as lattice compression; the reduction of the $a$-axis $(\sim 1 \%$ under $3 \mathrm{GPa})$ in $\mathrm{LaFeAsO}_{1-x} \mathrm{~F}_{x}$ is assumed to bring it close to a $\mathrm{LaFeAsO}_{1-x} \mathrm{H}_{x}$ lattice ${ }^{16}$. Then, a $1 \%$-reduction of $a$-axis for $\mathrm{LaFeAsO}_{1-x} \mathrm{H}_{x}$ draws its lattice close to that of $\mathrm{CeFeAsO}_{1-x} \mathrm{H}_{x}$ under an ambient pressure ${ }^{12}$.

Lanthanide cation substitution effect. Here we consider the relation between the two domes of the La-system and the domes of the other $L n$-1111 systems. To compare the temperature dependence of resistivity of $\mathrm{LaFeAsO}_{1-x} \mathrm{H}_{x}$ with that of other $L n-1111$ systems, we performed power-law fitting, $\rho=\rho_{0}+A T^{n}$ ( $\rho_{0}$ : residual resistivity) in the temperature range above $T_{\mathrm{c}}$ to $150 \mathrm{~K}$ (Supplementary Fig. S1). Figure $3 \mathrm{a}$ shows the relation between the exponents $n$ and $x$ for $L n \mathrm{FeAsO}_{1-x} \mathrm{H}_{x}(\mathrm{Ln}=\mathrm{La}, \mathrm{Ce}, \mathrm{Sm}$ and $\mathrm{Gd}$; The sample preparation and temperature dependence of electrical resistivity and volume magnetic susceptibility for newly found $\mathrm{GdFeAsO}_{1-x} \mathrm{H}_{x}$ 

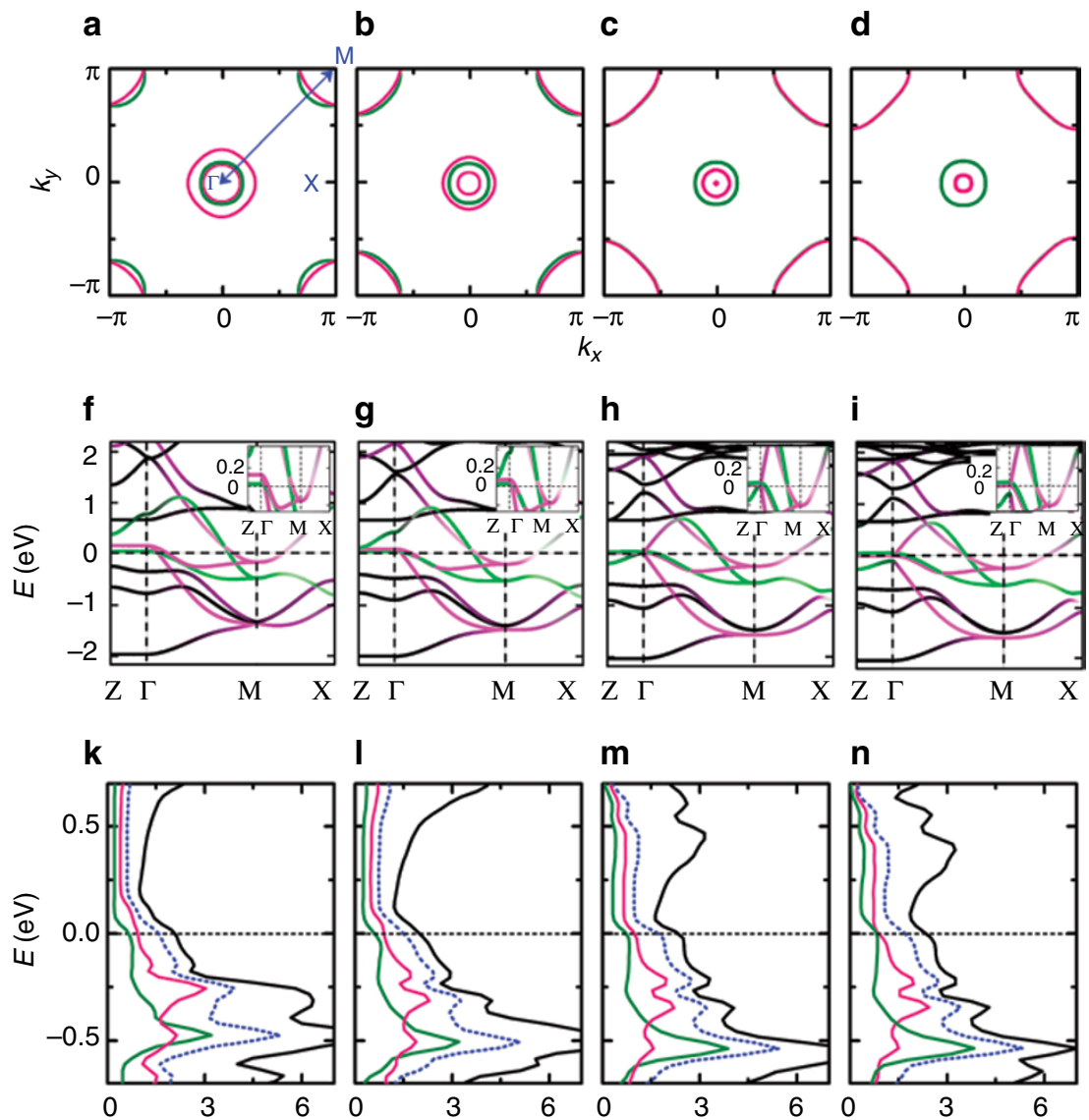

m

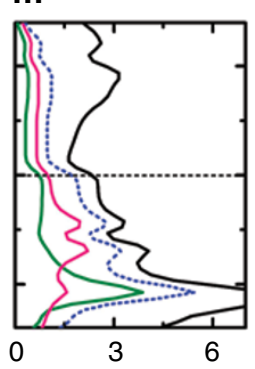

n

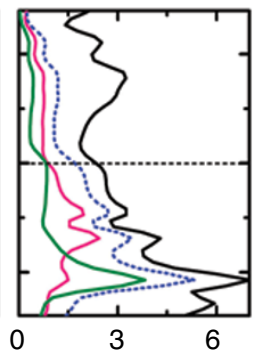

Density of states $\left(\mathrm{eV}^{-1}\right.$ f.u. $\left.{ }^{-1}\right)$ e
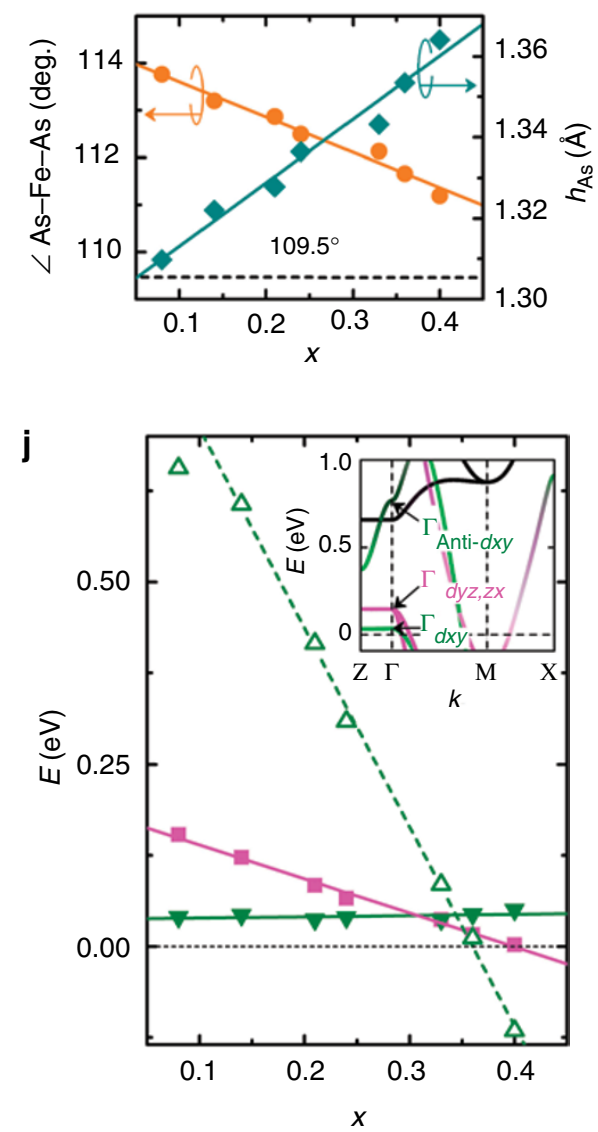

Figure 4 | Electronic structure of $\mathrm{LaFeAsO}_{\mathbf{1 - x}} \mathbf{H}_{\mathbf{x}}$ (a-d) Two-dimensional Fermi surface of LaFeAsO ${ }_{1-x} \mathrm{H}_{x}$ with $x=0.08$ (a), $0.21(\mathbf{b}), 0.36(\mathbf{c})$ and 0.40 (d). The blue arrow represents the nesting vector in the $(\pi \pi)$ direction. The contribution of Fe- $d_{x y}$ and $d_{y z, z x}$ orbitals are coloured green and pink, respectively ${ }^{21}$. (e) Hydrogen doping dependence of As-Fe-As angle in FeAs 4 tetrahedron (orange circles) and arsenic height $h_{\mathrm{As}}$ from the Fe plane (dark sky-blue diamonds). The angles and $h_{\mathrm{As}}$ are determined from Synchrotron $\mathrm{X}$-ray diffraction measurements at $20 \mathrm{~K}$. The solid lines are as a visual guide. The dashed line denotes the regular tetrahedron angle. (f-i) Band structures of $\mathrm{LaFeAsO}_{1-x} \mathrm{H}_{x}$ with $x=0.08(\mathbf{f}), 0.21$ ( $\left.\mathbf{g}\right), 0.36$ (h) and 0.40 (i). Insets show close-up views of the low energy region. The contribution of Fe- $d_{x y}$ and $d_{y z, z x}$ orbitals are coloured green and pink, respectively. (j) Variation in energy level of relevant Fe $3 d$ bands at $\Gamma$ point with $x$. The inset is the band structure of LaFeAsO ${ }_{0.92} \mathrm{H}_{0.08}$. The $\Gamma_{d x y}$ (filled green inverted triangles) and $\Gamma_{\text {anti- } d x y}$ (open green triangles) signify the bonding and anti-bonding states, respectively, for a bond primary composed of two Fe $d_{x y}$ orbitals in a unit cell. Also shown is the energy level of degenerate $d_{y x, z x}$ band ( $\Gamma_{d y z, z x}$ indicated by filled pink squares). The solid and dashed lines are as a visual guide. (k-n) Total DOS (solid black line) and partial DOS of $d_{x y}$ (solid green line) and $d_{y z, z x}$ (solid pink line) orbitals of $\mathrm{LaFeAsO}_{1-x} \mathrm{H}_{x}$ with $x=0.08(\mathbf{k}), 0.21$ (I), 0.36 $(\mathbf{m})$ and $0.40(\mathbf{n})$. The sum of the partial DOS of $d_{x y}$ and $d_{y z, z x}$ orbitals is also shown (dotted blue line).

are summarized in Supplementary Fig. S2 and in the Supplementary Methods.). Fermi liquid-like behaviour, $n=2$, is observed only in low- $x \mathrm{LaFeAsO}_{1-x} \mathrm{H}_{x}$, whereas non-Fermi liquid behaviuor, $n<2$, is observed for high- $x \mathrm{LaFeAsO}_{1-x} \mathrm{H}_{x}$ and for the entire range of $x$ in the other systems. Figure $3 \mathrm{~b}$ shows the plot of $T_{\mathrm{c}}$ versus exponent $n$ for the same systems. As $n$ approaches unit for each system, $T_{\mathrm{c}}$ becomes a maximum, indicating that this feature of the second dome in $\mathrm{LaFeAsO}_{1-x} \mathrm{H}_{x}$ is commonly seen for domes in other $\mathrm{Ln}$ 1111; that is, the first dome is unique to La-1111, whereas the second is universally to all four systems. Figure $3 \mathrm{c}-\mathrm{f}$ shows the $x$-variation in $T_{\mathrm{c}}$ for all four systems. The optimal $x$ in the $T_{\mathrm{c}}$ dome continuously shifts to lower $x$ when comparing $L n=\mathrm{La}$ through to $\mathrm{Gd}$.

\section{Discussion}

We have found an unusual two-dome structure in $T_{\mathcal{c}}$ for the LaFeAsO $\mathrm{O}_{1-x} \mathrm{H}_{x}$ system, the higher $T_{\mathrm{c}}$ dome being associated with a

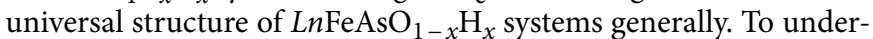
stand these dome structures, we calculated the electronic state of these materials using the crystal structures determined at $20 \mathrm{~K}$. The electron doping via substitution of $\mathrm{O}^{2-}$ sites with $\mathrm{H}^{-}$ion was modelled in virtual crystal approximation assuming hydrogen acts as a quasi-fluorine ion supplying an electron to the FeAs layer ${ }^{12}$, that is, the oxygen $(Z=8)$ sites were substituted for virtual atoms which have a fractional nuclear charge $(Z=8+x)$, where $x$ is hydrogen fraction. Figure $4 \mathrm{a}-\mathrm{d}$ shows the two-dimensional cross-sections of FS for the various doping levels. These compositions, $x=0.08,0.21$, 0.36 and 0.40 , correspond to the top of first dome, $T_{\mathrm{c}}$ valley, the top of the second dome and over-doping region, respectively. At $x=0.08$, the size of an outer $d_{x y}(x, y$ and $z$ coordination is given by the $\mathrm{Fe}$ square lattice) or inner $d_{y z, z x}$ hole pockets at the $\Gamma$ point is close to that of two electron pockets at the M point, indicating that nesting in the $(\pi \pi)$ direction between the hole and the electron pockets is strong. As $x$ increases, the nesting monotonically weakens because the hole pockets are gradually reduced but the electron pockets are expanded. It is pointed out that as the pnictogen height, $h_{\mathrm{Pn}}$, from the Fe plane increases, the $d_{x y}$ hole pocket is enlarged; nesting then becomes better ${ }^{10}$. In the present case, although $h_{\mathrm{As}}$ increases with $x$ as shown in Fig. $4 \mathrm{e}$, the size of the $d_{x y}$ hole pocket remains almost 
unchanged irrespective of $x$. This result may be understood by considering that expansion of $d_{x y}$ hole pocket by structural modification is cancelled by reduction due to the up-shift of Fermi level $\left(E_{\mathrm{F}}\right)$ by electron doping.

Nesting between hole and electron pockets is the most important glue in the spin fluctuation model ${ }^{8,9}$. The decrease in $T_{c}$ from $x=0.08$ through 0.21 may be understood as a reduction in spin fluctuations due to weakening of the nesting in a similar manner to $\mathrm{LaFeAsO}_{1-x} \mathrm{~F}_{x}$. It is, however, difficult to understand by the FS nesting that the experimental findings that $T_{\mathrm{c}}(x)$ increases over a wider dome range of $0.21<x<0.53$ and optimizes at $36 \mathrm{~K}$ around $x=0.36$.

Figure $4 \mathrm{f}-\mathrm{i}$ shows band structures near $E_{\mathrm{F}}$ for sample composition $x=0.08,0.21,0.36$ and 0.40 . As the unit cell contains two irons, there are ten bands around the $E_{\mathrm{F}}$ derived from bonding and antibonding of $3 d$ orbitals of the two neighbouring irons that cross the $E_{\mathrm{F}}$ around the $\Gamma$ and $\mathrm{M}$ points. The unoccupied bands move continuously lower with $x$. In particular, the bands derived from the anti-bonding orbital between the $d_{x y}$ orbitals, which we shall call the 'anti- $d_{x y}$ bands' hereafter, and the band derived from the degenerate $d_{y z, z x}$ bonding orbitals are lowered in energy and cross the bonding$d_{x y}$ band around $x=0.36$, forming degenerate states of $\mathrm{Fe} 3 d_{x y, y z, z x}$ three bands near $E_{\mathrm{F}}$ as seen in Fig. $4 \mathrm{j}$. After this triply-degenerate state is formed, the anti- $d_{x y}$ band and bonding $d_{y z, z x}$ band create a new band below $E_{\mathrm{F}}$ at $x=0.40$ by reconstruction (see inset of Fig. 4i). Note in Fig. 4e that the As-Fe-As angle of FeAs 4 tetrahedron is far from that of regular tetrahedron $\left(109.5^{\circ}\right)$ in the optimally doped region $(x=0.33-0.46)$. The band structures of $\mathrm{LaFeAsO}_{1-x} \mathrm{H}_{x}$ $(x=0.08,0.21,0.36$ and 0.40$)$ with only structural change are shown in Supplementary Fig. S3. Although the magnitude of the energy difference between these three bands becomes small with $x$, the band crossing is not caused only by the structural change, indicating that not only the change in local structure around iron but also asymmetric occupation of doped electrons in the bonding- $d_{y z, z x}, d_{x y}$ and anti-bonding- $d_{x y}$, affect the band shift. As the bonding $d_{y z, z x}$ and $d_{x y}$ bands are almost flat along the $\Gamma-Z$ direction, their band crossing at $x=0.36$ form a shoulder in the total density of states (DOS) at $E_{\mathrm{F}}$ (Fig. $4 \mathrm{~m}$ ), indicating an electronic instability of the system arising from degeneration of the $d_{x y}$ and $d_{y z, z x}$ bands. In such a situation, structural transitions, for instance band Jahn-Teller distortion, may occur to reduce the energy of the system. However, in the present results, any structural transition could not be observed at least down to $20 \mathrm{~K}$ in samples with $0.08 \leq x \leq 0.40$.

Table 1 summarizes the characteristics of each $T_{\mathrm{c}}$ dome described above. The primary question is what the origin is for the second dome, that is, the dome in the $L n-1111$ with higher $T_{\mathrm{c}}$. The resistivity above $T_{\mathrm{c}}$ changes from quadratic to linear dependence as $x$ approaches the top of the second dome region. As the nesting between hole and electron pockets monotonically is weakened with $x$, it is rationally considered that the contribution of FS nesting to the second $T_{\mathrm{c}}$ dome is not dominant. In addition, the calculated DOS shows the presence of a shoulder of the DOS $\left(E_{\mathrm{F}}\right)$ at $x=0.36$ related by the degeneracy of three bands derived from Fe- $d_{y z, \mathrm{zx}}$ and $d_{x y}$ orbitals. Given these results, the band degeneracy appears to have an important role in emergence of the second dome. For the iron-based superconductors, there is another pairing model derived from a large softening of a shear modulus observed near the tetragonal-orthorhombic transition of parent compounds ${ }^{17-19}$. This model tells that the Fe- $d$ orbitals are possible to order when their degeneracy in Fe- $d_{y z, z x}$ orbitals is removed at the structural transition and the fluctuations of this orbital ordering are shown capable of inducing superconductivity ${ }^{18,19}$. If we follow the orbital fluctuation model, the second dome and $T$-linear resistivity in the present system might be understood as results of electron pairing and carrier scattering by the fluctuations of the degenerated Fe- $d_{x y, y z, z x}$ orbitals, respectively.
Table 1 | Characteristics of two $T_{c}$ domes in LaFeAsO ${ }_{1-x} H_{x}$.

\begin{tabular}{lll} 
Dome & First & Second \\
\hline$x$ & $0.05 \leq x \leq 0.2$ & $0.2 \leq x \leq 0.5$ \\
Exponent, $n$ & $0.0 \leq n \leq 2.3$ & $0.7 \leq n \leq 2.0$ \\
$T_{c}$ max & $29 \mathrm{~K}$ & $36 \mathrm{~K}$ \\
$T_{\mathrm{c}}$ sensitivity to $x$ & High & Low \\
Under high pressure & Unified & Unified \\
FS nesting between hole and & Strong & Weak \\
electron pockets & & \\
$\operatorname{DOS}\left(E_{\mathrm{F}}\right)$ & No shoulder & Shoulder \\
\hline
\end{tabular}

Finally, we consider why the two-dome structure is only found in $\mathrm{LaFeAsO}_{1-x} \mathrm{H}_{x}$ and not (Ce-Gd)FeAsO $\mathrm{F}_{1-x} \mathrm{H}_{x}$. The degeneracy of three bands derived from $\mathrm{Fe}-3 d_{y z, z x}$ and $d_{x y}$ orbitals is realized when energy splitting between the $3 d_{y z, z x}$ and $d_{x y}$ bands mainly derived from distortion of $\mathrm{FeAs}_{4}$ tetrahedron is cancelled out by asymmetric occupation of doped electrons in the these three bands. The magnitude of the energy difference between these three bands becomes small on going from $\mathrm{La}$ ion to $\mathrm{Ce}-\mathrm{Gd}$ ions in $\mathrm{LnFeAsO}$ because the As-Fe-As angle of $\mathrm{FeAs}_{4}$ tetrahedron continuously approach to the angle $\left(109.5^{\circ}\right)$ of a regular tetrahedron as the lanthanide ion is changed from $\mathrm{La}\left(114^{\circ}\right)$ to $\mathrm{Gd}\left(110^{\circ}\right)^{20}$. In particular, this deviation in the La-system is rather large compared with the other system. Therefore, the threefold degeneracy in $(\mathrm{Ce}-\mathrm{Gd}) \mathrm{FeAsO}_{1-x} \mathrm{H}_{x}$ on electron doping is expected to occur in lower $x$ than that $(x=0.35)$ in $\mathrm{LaFeAsO}_{1-x} \mathrm{H}_{x}$. As a consequence, we think that the second dome primarily originating from the band degeneracy is separated from the first dome from FS nesting. The present discussion on orbital fluctuation model is based on DFT calculations. Further effort is required to confirm the validity of this idea on decisive experimental evidences such as angular-resolved photoemission and elastic shear modulus measurements using the single crystals.

\section{Methods}

Synthesis, structural and chemical analyses of $\mathrm{LaFeAsO}_{1-x} \mathrm{H}_{\mathbf{x}}$. As reported previously, $\mathrm{LaFeAsO}_{1-x} \mathrm{H}_{x}$ was synthesized by solid-state reactions using starting materials $\mathrm{La}_{2} \mathrm{O}_{3}, \mathrm{LaAs}, \mathrm{LaH}_{2}, \mathrm{FeAs}$ and $\mathrm{Fe}_{2} \mathrm{As}$ under high pressure ${ }^{11}$. The phase purity and structural parameters at room temperature were determined by powder $\mathrm{X}$-ray diffraction measurements with MoK $\alpha 1$ radiation. The structure parameters at low temperatures were determined by synchrotron $\mathrm{X}$-ray diffraction measurements at $20 \mathrm{~K}$ using the BL02B2 beam-line in the SPring-8, Japan. Hydrogen content in the synthesized samples was evaluated by thermogravimetric mass spectroscopy, and the chemical composition with the exception of hydrogen was determined with a wavelength-dispersive-type electron-probe microanalyzer.

Electric and magnetic measurement. Resistivity and magnetic susceptibility at ambient pressure were measured using a physical property measurement system with a vibrating sample magnetometer attachment. Electrical resistivity measurements under high pressure were performed by the dc four-probe method. Pressures were applied at room temperature and maintained using a piston-cylinder device. A liquid pressure-transmitting medium (Daphne oil 7373) was used to maintain hydrostatic conditions.

Density functional theory calculations. The electronic structure for $\mathrm{LaFeAsO}_{1-x}$ $\mathrm{H}_{x}$ was derived from non-spin-polarized DFT calculations using the WIEN2K $\operatorname{code}^{22}$ using the generalized gradient approximation Perdew-Burke-Ernzerhof functional ${ }^{23}$ and the full-potential linearized augmented plane wave plus localized orbitals method. To ensure convergence, the linearized augmented plane wave basis set was defined by the cutoff $R_{\mathrm{MT}} K_{\mathrm{MAX}}=9.0\left(R_{\mathrm{MT}}\right.$ : the smallest atomic sphere radius in the unit cell), with a mesh sampling of $15 \times 15 \times 9 k$ points in the Brillouin zone.

\section{References}

1. Kamihara, Y., Watanabe, T., Hirano, M. \& Hosono, H. Iron-based layered superconductor $\mathrm{La}\left[O_{1-x} \mathrm{~F}_{x}\right] \mathrm{FeAs}(x=0.05-0.12)$ with $T_{c}=26 \mathrm{~K}$. J. Am. Chem. Soc. 130, 3296-3297 (2008).

2. Rotter, M., Tegel, M. \& Johrendt, D. Superconductivity at $38 \mathrm{~K}$ in the iron arsenide $\left(\mathrm{Ba}_{1-x} \mathrm{~K}_{x}\right) \mathrm{Fe}_{2} \mathrm{As}_{2}$. Phys. Rev. Lett. 101, 107006 (2008). 
3. Wang, X. C. et al. The superconductivity at $18 \mathrm{~K}$ in LiFeAs system. Solid State Commun. 148, 538-540 (2008)

4. Zhu, X. et al. Transition of stoichiometric $\mathrm{Sr}_{2} \mathrm{VO}_{3} \mathrm{FeAs}$ to a superconducting state at 37.2 K. Phys. Rev. B 79, 220512 (2009).

5. Ren, Z. A. et al. Superconductivity at $55 \mathrm{~K}$ in iron-based F-doped layered quaternary compound $\mathrm{Sm}\left[\mathrm{O}_{1-x} \mathrm{~F}_{x}\right]$ FeAs. Chin. Phys. Lett. 25, 2215-2216 (2008).

6. de la Cruz, C. et al. Magnetic order close to superconductivity in the iron-based layered $\mathrm{LaO}_{1-x} \mathrm{~F}_{x} \mathrm{FeAs}$ systems. Nature 453, 899-902 (2008).

7. Nomura, T. et al. Crystallographic phase transition and high- $T_{\mathrm{c}}$ superconductivity in LaFeAsO: F. Supercond. Sci. Technol. 21, 125028 (2008).

8. Mazin, I. I., Singh, D. J., Johannes, M. D. \& Du, M. H. Unconventional superconductivity with a sign reversal in the order parameter of $\mathrm{LaFeAsO}_{1-x} \mathrm{~F}_{x}$. Phys. Rev. Lett. 101, 05700 (2008).

9. Kuroki, K. et al. Unconventional pairing originating from the disconnected fermi surfaces of superconducting $\mathrm{LaFeAsO}_{1-x} \mathrm{~F}_{x}$. Phys. Rev. Lett. 101, 087004 (2008).

10. Kuroki, K., Usui, H., Onari, S., Arita, R. \& Aoki, H. Pnictogen height as a possible switch between high- $T_{\mathrm{C}}$ nodeless and low- $T_{\mathrm{c}}$ nodal pairings in the iron-based superconductors. Phys. Rev. B 79, 224511 (2009).

11. Hanna, T. et al. Hydrogen in layered iron arsenides: indirect electron doping to induce superconductivity. Phys. Rev. B. 84, 024521 (2011).

12. Matsuishi, S. et al. Structural analysis and superconductivity of $\mathrm{CeFeAsO}_{1-x} \mathrm{H}_{x}$. Phys. Rev. B. 85, 014514 (2012).

13. Imada, M., Fujimori, A. \& Tokura, Y. Metal-insulator transitions. Rev. Mod. Phys. 70, 1039 (1998)

14. Takahashi, H. et al. Superconductivity at $43 \mathrm{~K}$ in an iron-based layered compound $\mathrm{LaO}_{1-x} \mathrm{~F}_{x} \mathrm{FeAs}$. Nature 453, 376-378 (2008)

15. Hess, C. et al. The intrinsic electronic phase diagram of iron-oxypnictide superconductors. Europhys. Lett. 87, 17005 (2009).

16. Takahashi, H. et al. High-pressure studies on superconducting iron-based $\mathrm{LaFeAsO}_{1-x} \mathrm{~F}_{x}$, LaFePO and $\mathrm{SrFe}_{2} \mathrm{As}_{2}$. J. Phys. Soc. Jpn. 77, 78 (2008).

17. Yoshizawa, M. et al. Structural quantum criticality and superconductivity in iron-based superconductor $\mathrm{Ba}\left(\mathrm{Fe}_{1-x} \mathrm{Co}_{x}\right)_{2} \mathrm{As}_{2}$. J. Phys. Soc. Jpn. 81, 024604 (2012).

18. Kontani, H. \& Onari, S. Orbital-fluctuation-mediated superconductivity in iron pnictides: analysis of the five-orbital hubbard-holstein model. Phys. Rev. Lett. 104, 157001 (2010).

19. Yanagi, Y., Yamakawa, Y., Adachi, N. \& Ōno, Y. Orbital order, structural transition, and superconductivity in iron pnictides. J. Phys. Soc. Jpn. 79, 123707 (2010).
20. Wang, P., Stadnik, Z. M., Wang, C., Cao, G. H. \& Xu, Z. A. Transport, magnetic, and ${ }^{57} \mathrm{Fe}$ and ${ }^{155} \mathrm{Gd}$ Mössbauer spectroscopic properties of GdFeAsO and the slightly overdoped superconductor $\mathrm{Gd}_{0.84} \mathrm{Th}_{0.16} \mathrm{FeAsO}$. J. Phys.:Condens. Matt. 22, 145701 (2010).

21. Haverkort, M. W., Elfimov, I. S., Tjeng, L. H., Sawatzky, G. A. \& Damascelli, A. Strong spin-orbit coupling effects on the fermi surface of $\mathrm{Sr}_{2} \mathrm{RuO}_{4}$ and $\mathrm{Sr}_{2} \mathrm{RhO}_{4}$. Phys. Rev. Lett. 101, 026406 (2008).

22. Blaha, P. et al. An Augmented Plane Wave and Local Orbitals Program for Calculating Crystal Properties, Technical University of Wien, Vienna( 2001).

23. Perdew, J. P., Burke, K. \& Ernzerhof, M. Generalized gradient approximation made simple. Phys. Rev. Lett. 77, 3865 (1996); 78, 1396(E) (1997).

\section{Acknowledgements}

We thank Professor H. Fukuyama of Tokyo university of Science for discussions. This research was supported by the Japan Society for the Promotion of Science (JSPS) through the FIRST program, initiated by the CSTP. The synchrotron radiation experiments were performed at the BL02B2 of SPring-8 with the approval of the Japan Synchrotron Radiation Research Institute (JASRI; proposal no. 2011A1142).

\section{Author contributions}

H.H. and S.M. planned the research. S.I., T.H. and Y.M. performed the high-pressure synthesis. S.I. performed measurement. H.S. and S.W.K. carried out high-pressure resistivity measurement. S.I., J.E.K. and M.T. performed Synchrotron X-ray diffraction measurements. S.I. and S.M. performed DFT calculations. H.H. and S.I. and S.M. discussed the results and wrote the manuscript.

\section{Additional information}

Supplementary Information accompanies this paper at http://www.nature.com/ naturecommunications

Competing financial interests: The authors declare no competing financial interests.

Reprints and permission information is available online at http://npg.nature.com/ reprintsandpermissions/

How to cite this article: Iimura, $\mathrm{S}$ et al. Two-dome structure in electron-doped iron arsenide superconductors. Nat. Commun. 3:943 doi: 10.1038/ncomms1913 (2012).

License: This work is licensed under a Creative Commons Attribution-NonCommercialNoDerivative Works 3.0 Unported License. To view a copy of this license, visit http:// creativecommons.org/licenses/by-nc-nd/3.0/ 


\section{Corrigendum: Two-dome structure in electron-doped iron arsenide superconductors}

Soshi limura, Satoru Matsuishi, Hikaru Sato, Taku Hanna, Yoshinori Muraba, Sung Wng Kim, Jung Eun Kim, Masaki Takata \& Hideo Hosono

Nature Communications 3:943 doi: 10.1038/ncomms1913 (2012); Published 10 Jul 2012; Updated 20 Dec 2013

The original version of this Article contained a typographical error in the spelling of the author Satoru Matsuishi, which was incorrectly given as Satoru Matuishi. This has now been corrected in both the PDF and HTML versions of the Article. 